\title{
Justification of strategies for agricultural countermeasures in the long term after the Chernobyl accident based on a cost-benefit analysis
}

\author{
A.V. Panov, S.V. Fesenko, A.Yu. Pakhomov and R.M. Alexakhin
}

Russian Institute of Agricultural Radiology and Agroecology, 249030 Obninsk, Russia

\begin{abstract}
In the long term after the Chernobyl accident the introduction of systems of countermeasures in agriculture must be based on the optimization principle. To implement this principle, a concept was used of evaluation of the effectiveness of countermeasures based on a cost-benefit analysis. Countermeasure options were developed separately for collective and private sectors of rural settlements. For each type of farming a range of countermeasures were defined and the optimal ones were identified. The effectiveness of countermeasures was estimated on the basis of integral criteria: cost of averted collective dose (1 man-Sv), overall costs needed for countermeasures introduction and time for fulfilling legal regulations. Based on the most effective countermeasures, optimal combinations (strategies) were developed. An assessment was given of the effectiveness of countermeasures aimed at reducing the radionuclide content in animal products from collective farms and lowering doses to rural residents affected by the Chernobyl accident, based on a comparative cost-benefit analysis. To optimize costs of the rehabilitation of agricultural lands, the most optimal in terms of meeting the standards strategy was determined, which is an address application of countermeasures.
\end{abstract}

\section{INTRODUCTION}

Currently, dose burdens to the population living in the Chernobyl affected area of the Russian Federation where intervention is needed ranges between 1 and $5 \mathrm{mSv} \mathrm{y}^{-1}$. According to the law "On the radiation safety", the introduction of countermeasures in such areas is to be based on the optimization principle [1]. To realize this principle, a concept was used for the assessment of countermeasures effectiveness based on the cost-benefit analysis [2].

In previous investigations a methodology has been developed for the assessment of countermeasures effectiveness in collective farms and rural settlements, criteria and stages of justification of the optimum use of countermeasures have been determined. The effectiveness of some countermeasures as well as their combinations for different contamination zones has been analyzed [3]. The derived results make it feasible to switch to the justification of optimum strategies of farming in collective and private farms on the basis of a comparative analysis of the ratio of countermeasures cost and benefit in the form of collective dose saving.

The effectiveness of countermeasures has been evaluated based on the integral criteria: cost of averted collective dose (1 man-Sv), total costs necessary for countermeasures implementation and times needed to meet the standards (the law "On the radiation safety" and SanPin standards on ${ }^{137} \mathrm{Cs}$ content in farm products $[1,4])$.

Assessment of the effectiveness consisted of three steps. At the first step, for each type of farming, a range of countermeasures was identified and averted collective doses due to their application were calculated. At the second stage total costs were estimated necessary for applying of countermeasures, with all the countermeasures being arranged by this criterion. The third step 
included analysis of the ratio of averted collective dose cost (1 man-Sv) and total costs of countermeasures. Evidently that the most optimum may be recognized the countermeasures with the lowest cost of 1 man-Sv and total costs.

\section{ASSESSMENT OF THE EFFECTIVENESS OF INDIVIDUAL COUNTERMEASURES}

The most effective measure for reducing milk contamination in the collective sector is the use of ferrocyn containing compounds (FCC). The cost of 1 man-Sv when this countermeasure is generally applied over the territory where milk contamination is above the SanPin standards will amount to about 1 thousand EURO at a total cost of 2.6 million EURO (Fig. 1-A). The effectiveness of the radical improvement (RI) of lands is higher than that of silage ration for cows in the indoor period and their feeding by the "green conveyer" type in the pasture period (SIL/GC). Even though the cost of radical improvement is some 400 thousand EURO higher, the cost of 1 man-Sv is 2.2 times lower.
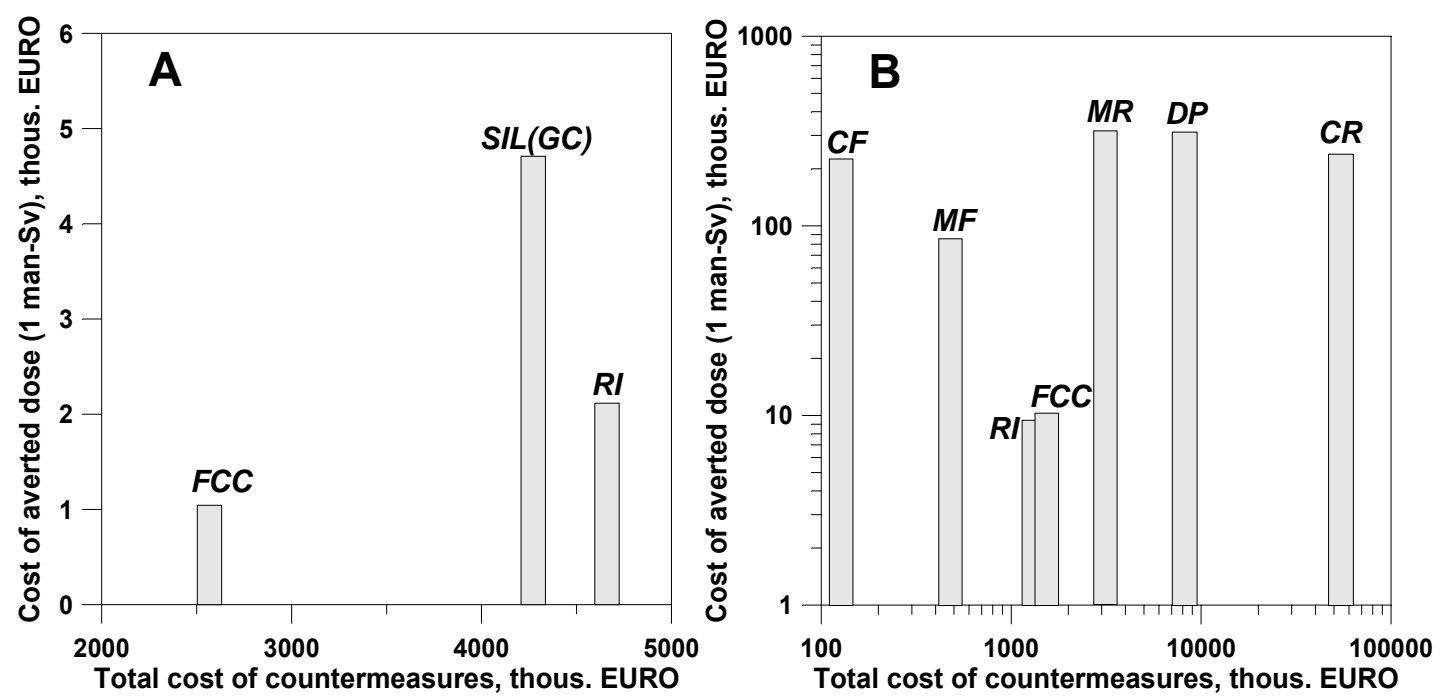

Figure 1. Cost of averted collective dose versus total cost in the event of individual countermeasure introduction in the collective (A) and private (B) sector.

The most effective countermeasures in the private sector are radical improvement of haylands and pastures and FCC application in all the areas where the dose limits $\left(1 \mathrm{mSv} \mathrm{y}^{-1}\right)$ are exceeded. These measures are similar in both cost of averted dose (9.98 and 10.42 thousand EURO) and total costs (1.42 and 1.48 million EURO, Fig. 1-B). The use of mineral fertilizers under potato (MF) and "clean" fodders for cows (CF) over three months before slaughtering rank, respectively, the third and fourth places. Though the cost of mineral fertilizing is higher compared with the use of "clean" feeds by 355 thousand EURO, the cost of 1 man-Sv is 2.6 times lower (86.1 and 223.8 thousand EURO, respectively). Since the cost of the two above measures is noticeably lower than that of radical improvement of lands and FCC application, these may be recommended in the most affected regions with increased ${ }^{137} \mathrm{Cs}$ content in beef and potato. The three least effective countermeasures are restrictions on collection and consumption of mushrooms (MR), decontamination of private farms (DP) and restrictions on private dairy cows (CR). The cost of these countermeasures in the entire contaminated area with an annual mean dose of exposure of the rural residents above $1 \mathrm{mSv}$ varies from 3 to 52.7 million EURO, and the cost of 1 man-Sv from 240 to 320 thousand EURO.

Hence, should in the early period after the Chernobyl accident the restrictive and decontamination measures were justified, in the long term these are not expedient in all rural settlements where doses to the residents are above the limits. Thus, restrictions on gathering and consuming mushrooms should 
be imposed only in the most critical rural settlements located near forest where the contribution of this product to the internal dose is very high. Decontamination measures are necessary only there where the contribution of external exposure to the total dose burden is markedly above that of internal irradiation and where it is impossible to achieve dose reduction by no other methods. Therefore, the decision on the use of such extreme measures must be made on the basis of a radiologic-economic analysis of the situation in the most contaminated regions.

\section{ASSESSMENT OF THE EFFECTIVENESS OF COUNTERMEASURES STRATEGIES}

Based on the classification of farms and settlements, strategies of countermeasures have been developed that ensure production of stuffs which totally meet the SanPin standards (the collective sector) and reduction of the population exposure below $1 \mathrm{mSv} \mathrm{y}^{-1}$ (the private sector).

For the rehabilitation of farms six strategies have been considered.

Strategy A. Application of countermeasures in the 1999-2002 scales.

Strategy B. Application of Prussian Blue in periods when an increased content of radionuclides is noted in products (critical periods) in all the farms where risk of SanPin excess is above 5\%.

Strategy C. Application of Prussian Blue on necessary scales.

Strategy D. Feeding of farm animals in the indoor period with a silage ration and in the pasture period by a "green conveyer" method.

Strategy E. A system of organizational and agrotechnical measures for every farm taking into account local features of animal farming.

Strategy F. Address rehabilitation.

For the rehabilitation of rural settlements also six strategies have been considered (Table 1).

Strategy A. Application of countermeasures in the 1999-2002 scales.

Strategy B. The strategy based on radical improvement of lands.

Strategy C. Combined application of radical improvement and Prussian Blue.

Strategy D. Address application of countermeasures and step-by-step rehabilitation of settlements with high doses to the residents.

Strategy E. Address and step-by-step rehabilitation without decontamination of the settlements with high doses to the residents.

Strategy F. Address application of countermeasures and introduction (continuation) of restrictions on private cattle keeping and decontamination of settlements with high doses to the population.

Table 1. Characteristics of countermeasures strategy for collective and private sector.

\begin{tabular}{|c|c|c|c|}
\hline Strategy & Total cost, thous. EURO & $\begin{array}{c}\text { Cost of averted dose } \\
\text { (1 man-Sv), thous. EURO }\end{array}$ & $\begin{array}{c}\text { Times (year) needed to } \\
\text { meet the standards }\end{array}$ \\
\hline \multicolumn{3}{|c|}{ Collective sector } \\
\hline A & 517 & 0.7 & 2026 \\
B & 633 & 0.5 & 2026 \\
C & 2568 & 1.0 & 2014 \\
D & 4272 & 4.7 & 2026 \\
E & 4661 & 2.1 & 2014 \\
F & 4733 & 2.0 & 2005 \\
\hline \multicolumn{4}{|c|}{ Private sector } \\
\hline A & 1442 & 12.9 & 2048 \\
B & 1422 & 9.98 & 2056 \\
C & 2906 & 15.9 & 2044 \\
D & 23996 & 101.4 & 2036 \\
E & 16131 & 76.1 & 2036 \\
F & 88900 & 371.3 & \\
\hline
\end{tabular}


The strategies of countermeasures application on the current scales in the collective sector (A) and FCC application in time periods when milk contamination is above the adopted standards (B) are alike in both costs of their introduction (517 and 633 thousand EURO) and cost of averted dose ( 0.7 and 0.5 thousand EURO). This indicates that currently countermeasures are restricted to selective application of FCC in some time periods and not in all the farms. Such a policy of countermeasures at one would think low costs will prevent achieving compliance with SanPin in the nearest time (deadline - no earlier than 2026). Therefore, strategies $\mathbf{A}$ and $\mathbf{B}$ cannot be considered as currently effective and can be recommended for use only in the event of negative development of the economic situation in the country. Low in effectiveness is strategy $\mathbf{D}$ based on animal feeding with a silage-haylage diet in the indoor and "green conveyer" in the pasture period. With this strategy the production of stuffs in accordance with the standards will only be possible by 2026. Taking into account both the highest cost of 1 man-Sv and total costs of this strategy, option $\mathbf{D}$ is considered to be low effective at the present time (Fig. 2-A).

The option $\mathbf{C}$ based on the use of FCC on necessary scales is more (4-5-fold) expensive than strategies A and B, however it will help to achieve the SanPin values much sooner, by 2014. Rather effective in terms of achieving the standard values is strategy $\mathbf{E}$ based on radical improvement of haylands and pastures. Despite the fact that the $\mathbf{C}$ strategy cost is $55 \%$ of the $\mathbf{E}$ option cost, one should take into account when comparing the two options an additional profit possibly resulting from the productivity increase of the fodder lands. The quickest transfer to the production of milk that totally meets the SanPin standards is achievable with the strategy of address rehabilitation (F), as early as by 2005; however this strategy is most expensive, albeit the cost of 1 man-Sv is low. A comparison of the most expensive strategies that include wide application of radical improvement $(\mathbf{E}$ and $\mathbf{F})$ shows that the use of other measures on the background of radical improvement must be strongly justified, since rational application of countermeasures increases their effectiveness and promotes achievement of ${ }^{137} \mathrm{Cs}$ levels in products in compliance with the standards at the earliest possible date. Overall, it should be stressed that all the strategies for the collective sector by the cost of 1 man-Sv are within the effectiveness scope recommended by the ICRP.
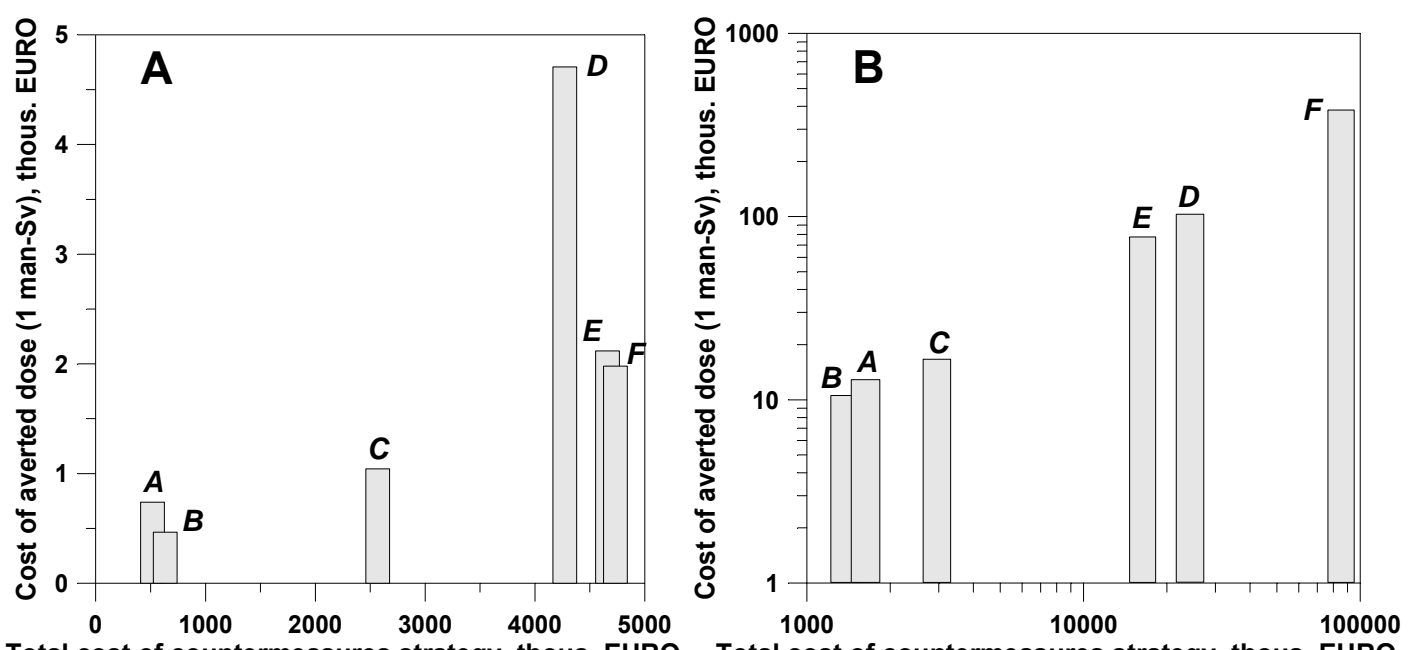

Total cost of countermeasures strategy, thous. EURO Total cost of countermeasures strategy, thous. EURO

Figure 2. Cost of averted collective dose versus total costs of different strategies of protective and rehabilitative measures in the collective (A) and private (B) sector.

All the recommended strategies for the private sector may be divided into two groups. The first one includes those based on the application of agricultural measures, namely introduction of countermeasures on the current scales (strategy A), radical improvement of lands on necessary scales (strategy B) and 
radical improvement plus FCC application (strategy $\mathbf{C}$ ). The costs of these countermeasures ranges within 1.4 and 2.9 million EURO and cost of 1 man-Sv from 10 to 16 thousand EURO, which is well within the effectiveness criteria recommended by the ICRP (Fig. 2-B). Measures currently being applied (strategy A) by the cost criterion rank between strategies $\mathbf{B}$ and $\mathbf{C}$ with a shift towards $\mathbf{B}$. This indicates that now in the rural settlements that need rehabilitation radical improvement of lands is adequately applied and in the most critical settlements FCC are applied. One, however, cannot but infer that application of agricultural measures alone does not solve the problem of rehabilitation of the rural settlements. In most affected, the fulfillment of the law "On the radiation safety" with the application of strategies A, B or C will not occur earlier than 2044 and at worst by 2056. The address rehabilitation without decontamination of the territory of the most contaminated settlements (strategy $\mathbf{E}$ ) is far less effective compared to strategy $\mathbf{C}$. By the time of the law fulfillment the two strategies coincide, whereas the introduction cost of strategy $\mathbf{E}$ is 5.6 times higher than $\mathbf{C}$ and cost of 1 man-Sv is also 4.8 times higher. A distinctive feature of the most expensive strategies ( $\mathbf{D}$ and $\mathbf{F}$ ) is the maximum possible fulfillment of the law "On the radiation safety" (by 2036). However, the address strategy of countermeasures that includes a step-by-step rehabilitation of the most critical rural settlements (strategy D) is much more effective than imposition in these settlements of restrictions on private cattle management during the entire period when this measure is necessary (strategy $\mathbf{F}$ ). By the total cost and cost of 1 man-Sv, strategy $\mathbf{D}$ is 3.7 times more efficient than strategy $\mathbf{F}$.

\section{ANALYSIS OF THE COST OF 1 MAN-SV FOR DIFFERENT COUNTERMEASURE STRATEGIES}

By definition, the cost of 1 man-Sv averted due to the application of countermeasures depends on the averted dose value and the resources spent. Evidently that when one or another measure (or their combination) is generally applied, initially some reduction in this parameter must be observed, and with time the growth. This is because the resources being spent for the application of countermeasures will remain the same and the effect in reducing exposure doses or products contamination will be declining due to natural ${ }^{137} \mathrm{Cs}$ decay.
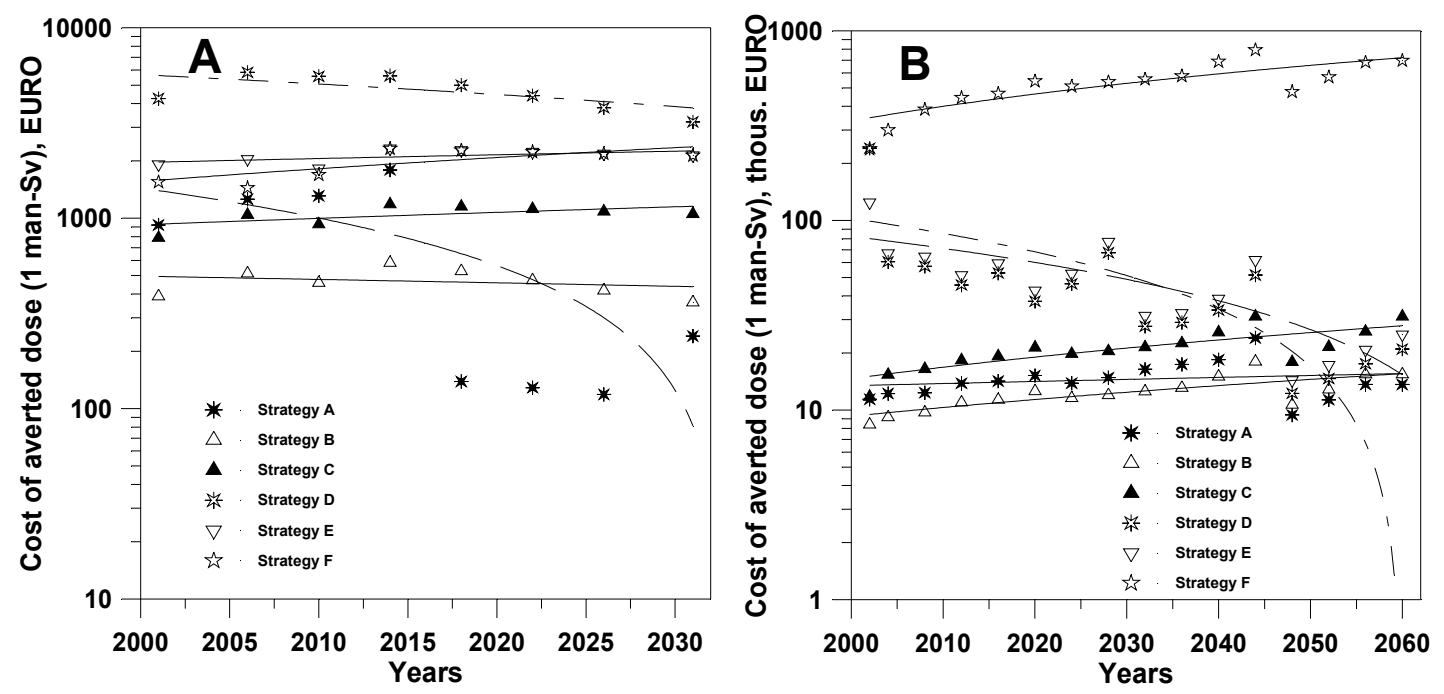

Figure 3. Dynamics of variations in the cost of collective dose reduction by 1 man-Sv for different countermeasures in the collective (A) and private (B) sector. 
In the collective sector (Fig. 3-A), when strategy $\mathbf{A}$ is used, the cost of 1 man-Sv first increases (from 0.9 to 1.8 thousand EURO) and since 2014 drops abruptly (up to 0.14-0.24 thousand EURO by 2018-2030). It means that the effectiveness of the current policy of selective FCC application will be reducing up to 2015 inclusive, following which risks of the SanPin excess will decrease to an extent that the production of milk in the collective sector in compliance with the standards can be maintained by FCC application in the most critical farms. The B strategy effectiveness does not practically change with time, i.e. it is applicable even in the longest term after the accident. For strategies $\mathbf{C}, \mathbf{E}$ and $\mathbf{F}$ a regular growth with time of the cost of 1 man-Sv is observed (from 10 to $30 \%$ over 30 years), which is connected with gradual decrease in their effect because of natural biochemical processes related to ${ }^{137} \mathrm{Cs}$ decay. Changes in the cost of 1 man-Sv with the use of strategy $\mathbf{D}$ is of a more complex character and is to a certain extent similar with variations in this parameter when strategy A is applied. Up to 2014 growth (from 4.3 to 5.6 thousand EURO) in this parameter is observed, then drop to 3.2 thousand EURO by 2030. Overall, a decrease in the cost of averted dose will amount to around $25 \%$. Considering that the cost of 1 man-Sv for this strategy is maximum, a possibility of its application cannot be viewed earlier than in 2014 and only in some farms.

In the private sector (Fig. 3-B), in all the strategies except for $\mathbf{D}$ and $\mathbf{E}$, there is growth in the cost of the averted dose with time. This is because strategies $\mathbf{A}, \mathbf{B}$ and $\mathbf{C}$ based on the use of agricultural countermeasures imply their introduction throughout the entire area on a full scale. Strategy $\mathbf{F}$ includes restriction on private cattle management in the most contaminated settlements for the whole time period while necessary. However, while for the agricultural countermeasures the growth will range from 1.2 to 2.5 times, for strategy $\mathbf{F}$ the cost of 1 man-Sv will increase by a factor of 3, which stresses once again an extreme ineffectiveness of continuing restrictive measures for a long time. The cost of 1 man-Sv when applying strategies $\mathbf{D}$ and $\mathbf{E}$ is declining with time, since these are based on a differential application of countermeasures on a necessary scale for each groups of settlement identified. This decline is especially observed in strategy D (12-fold), because the maximum expenses fall within the first year and are connected with decontamination of households. In strategy $\mathbf{D}$ (the same minus decontamination), such a decrease in the cost of 1 man-Sv is observed in a lesser extent (5-fold).

Assessment of the effectiveness of settlement rehabilitation strategies in terms of the ICRP recommendations (10-20 thousand US \$) shows that by this criterium effective are only strategies A and B. Strategy C becomes ineffective since 2024-2028, because the cost of 1 man-Sv since that time will be in excess of 20 thousand EURO. Strategies $\mathbf{D}$ and $\mathbf{E}$ become ineffective since 2048 and strategy F cannot be considered as effective because the averted dose cost in the event of its application exceeds by factors 10 to 35 the upper limit of the effectiveness recommended by the ICRP.

\section{CONCLUSION}

Hence, the data presented demonstrate that the existing capabilities for application of a complex of countermeasures aimed at reducing contamination of animal products and annual doses to the population provide great opportunities to achieve contamination levels that meet the SanPin Standard and the law "On the radiation safety of the population". The situation in the private sector of agriculture is more critical than on collective farms. This is evident from higher costs of the introduction of protective and rehabilitative measures and cost of possible averted doses due to their application, and consequently, the difference in the time of Standards achievement for the most effective countermeasures. Therefore, a return as soon as possible of the affected regions to conventional conditions of farming due to the introduction of some or other strategies will be dependent on the resources allocated for their implementation. 


\section{References}

[1] Federal law “On the radiation safety of the population”. FL No. 3, 09.01.96.

[2] Optimization of radiation protection based on the cost-benefit analysis. ICRP Publication 37. M: Energoatomizdat, 1985. 95 p.

[3] Fesenko S.V., Pakhomov A.Yu., Panov A.V. and Sanzharova N.I. "Identification of optimal countermeasures strategies in agriculture in the long term after the ChNPP accident”, ECORAD 2001: The radioecology-ecotoxicology of continental and estuarine environments: Proc. Intern. congress. France, 3-7 September, 2001. V. 1 / Ed. by F. Brechignac. Radioprotection. RadioprotectionColloques 2002. V. 37, C 1. P. 109-114.

[4] Hygienic codes for safety and nutrition value of foodstuffs: 2.3.2. Raw foods and foodstuffs. SanPin 2.3.2.1078-01. M.: Ministry of Health of Russia, 2002. 164 p. 